\title{
The bifurcation analysis and optimal feedback mechanism for an SIS epidemic model on networks
}

\author{
Lijuan Chen ${ }^{1 *} \mathbb{D}$, Shouying Huang ${ }^{1}$, Fengde Chen ${ }^{1}$ and Mingjian Fu'
}

"Correspondence:

chenlijuan@fzu.edu.cn

${ }^{1}$ College of Mathematics and

Computer Science, Fuzhou

University, Fuzhou, China

\section{Springer}

\begin{abstract}
It is well known that the feedback mechanism or the individual's intuitive response to the epidemic can have a vital effect on the disease's spreading. In this paper, we investigate the bifurcation behavior and the optimal feedback mechanism for an SIS epidemic model on heterogeneous networks. Firstly, we present the bifurcation analysis when the basic reproduction number is equal to unity. The direction of bifurcation is also determined. Secondly, different from the constant coefficient in the existing literature, we incorporate a time-varying feedback mechanism coefficient. This is more reasonable since the initiative response of people is constantly changing during different process of disease prevalence. We analyze the optimal feedback mechanism for the SIS epidemic network model by applying the optimal control theory. The existence and uniqueness of the optimal control strategy are obtained. Finally, a numerical example is presented to verify the efficiency of the obtained results. How the topology of the network affects the optimal feedback mechanism is also discussed.
\end{abstract}

Keywords: Complex networks; Epidemic dynamics; Feedback mechanism; Bifurcation; Optimal control

\section{Introduction}

In order to describe the effect of contact heterogeneity in a population, people have witnessed an increasing interest in epidemic system on networks [1-5]. Generally, the disease can spread through the interaction among individuals in a population. One can regard the above interaction as a network. Here the node represents the individual and each edge indicates the interaction among the individuals. In 2001, Pastor-Satorras and Vespignani [6] proposed a network-based SIS model to analyze the epidemic spreading. Subsequently, lots of epidemic models on networks, such as SIS [7-11], SIR [12, 13], SIRS [14, 15], WSIS [16], and SIQRS model [17], have been proposed to understand the transmission of infectious disease. In addition, the fractional SIR model, the epidemic model with distributed delay, the stochastic epidemic model, and the discrete-time epidemic model on complex networks were investigated in $[13,18,19]$, and [20], respectively.

(c) The Author(s) 2019. This article is licensed under a Creative Commons Attribution 4.0 International License, which permits use sharing, adaptation, distribution and reproduction in any medium or format, as long as you give appropriate credit to the original author(s) and the source, provide a link to the Creative Commons licence, and indicate if changes were made. The images or other third party material in this article are included in the article's Creative Commons licence, unless indicated otherwise in a credit line to the material. If material is not included in the article's Creative Commons licence and your intended use is not permitted by statutory regulation or exceeds the permitted use, you will need to obtain permission directly from the copyright holder. To view a copy of this licence, visit http://creativecommons.org/licenses/by/4.0/. 
As we know, most of the above literature works pay their attention to finding a threshold value $R_{0}$ which is vital to the epidemic spreading. In other words, most of the authors discuss the disease-free equilibrium and the endemic equilibrium when $R_{0}<1$ and $R_{0}>1$. For example, Zhang and Sun [7] investigate the following SIS epidemic model:

$$
\left\{\begin{array}{l}
\frac{d S_{k}(t)}{d t}=\delta-\delta S_{k}(t)-\lambda k(1-\beta \Theta(t)) S_{k}(t) \Theta(t)+\omega I_{k}(t), \\
\frac{d I_{k}(t)}{d t}=\lambda k(1-\beta \Theta(t)) S_{k}(t) \Theta(t)-(\omega+d) I_{k}(t), \quad k=1,2, \ldots, n .
\end{array}\right.
$$

Here, $S_{k}(t), I_{k}(t)$ represent the relative densities of susceptible and infected nodes with degree $k$ at time $t$ respectively. $\Theta(t)=\frac{1}{\langle k\rangle} \sum_{k=1}^{n} k P(k) I_{k}(t)$ is the probability that any given link points to the infected and $\langle k\rangle=\sum_{k=1}^{n} k P(k)$ is the average degree. $n$ is the maximum degree number. Also, $\delta$ is the natural birth rate, $d$ is the death rate, $\lambda$ is the transmission rate, and $\omega$ is the recovery rate. $\beta$ is "fear factor" which shows the fear degree when the individual faces up to the disease spread. As was pointed out in [7], one can assume that the birth rate equals the death rate, i.e., $\delta=d$. Let $R_{0}=\frac{\lambda\left\langle k^{2}\right\rangle}{(\delta+\omega)\langle k\rangle}$. The authors in [7] have obtained the results as follows.

Theorem A When $R_{0}<1$, the disease-free equilibrium is globally asymptotically stable.

Theorem B When $R_{0}>1$, the epidemic equilibrium is asymptotically stable.

Recently, Huang and Li [21] firstly proposed a network-based SIS epidemic model with a saturated treatment function and the existence of a backward bifurcation was investigated. Consequently, Li and Yousef in [22] presented the bifurcation analysis of a network-based SIR epidemic model with saturated treatment function. Motivated by [21] and [22], one can naturally propose the questions: What about $R_{0}=1$ for system (1.1)? Does there exist the bifurcation phenomenon? We regard the corresponding bifurcation analysis as an important study in epidemiology which motivates us to consider this critical case $R_{0}=1$.

At the same time, we notice that most of the above mentioned papers emphasize the qualitative analysis such as seeking the so-called basic reproduction number and then discussing the stability of equilibria and periodic orbits. Actually, another important way to analyze the epidemic spreading is the optimal control theory which pays attention to presenting an optimal strategy to deal with the disease outbreak. For the epidemic on heterogeneous networks, in [14] and [15], we firstly succeeded in studying the optimal control problem for an SIRS epidemic model on scale-free networks. By using the Pontryagin minimum principle, we $[14,15]$ found out an optimal strategy to minimize the total number of the infected and the cost associated with vaccination and treatment. Subsequently, there exist a large number of works that pay attention to the corresponding optimal control problem for the network-based epidemic model. One can see the details for this aspect in [23-27]. We notice that most of the above literature works consider the optimal strategy such as vaccination, quarantine, and treatment. As we know, once an epidemic disease outbreaks, people will be more cautious and consequently will reduce contacts with other people. In other words, people's initiative response to the disease should be considered when diseases prevail. Obviously, the feedback mechanism can change the contact among the individuals, which produces a new network topology structure. For example, in [7], the coefficient $\beta$ in system (1.1) can be called "fear factor" or "feedback parameter". Undoubtedly, it is indispensable to consider the influence of feedback mechanisms on 
disease transmission. Recently, lots of papers have investigated this aspect. In [28], a new model with a generalized feedback mechanism in weighted networks was investigated. In [29], a modified SIS network model with infective medium and feedback mechanism was presented. In [30], the authors obtained that the endemic equilibrium is globally asymptotically stable if the feedback parameter is sufficiently large or if the basic reproductive number belongs to a special interval. We notice that the feedback mechanism in [7, 29], and [30] is reflected by introducing a constant coefficient. However, we think that the initiative response of people is constantly changing and it is more reasonable to introduce the time-varying feedback parameter. If so, one may naturally propose an important and interesting question: what is the optimal feedback parameter or the optimal fear factor? As is well known, the public reaction to disease mostly depends on media coverage. In detail, inadequate media coverage can lead to a lack of awareness of the disease so that the disease is not easily controlled. However, the media's excessive reporting on the disease may cause panic in the whole society. In other words, how should the media reports present the disease properly? To the best of our knowledge, there exist few results that focus on the optimal analysis for the feedback coefficient, which can be regarded as an important factor when considering the transmission in a population. Motivated by the above, in this paper we propose an optimal control problem and find out the optimal feedback mechanism which can describe the initiative response to the disease.

Thus far, to the best of the authors' knowledge, this paper is the first work that addresses the problem about the bifurcation phenomenon and the optimal feedback mechanism for an SIS epidemic model on networks. The detailed contribution is in two aspects. One is to discuss the bifurcation phenomenon for the network-based epidemic model. The other is to propose the optimal feedback mechanism problem. We believe that this study will present the effect of the media coverage or the people's initiative response to the disease. The results can help the media to report the epidemic spreading suitably and then guide the individual to have effective protection when the disease outbreaks.

The remainder of this paper is organized as follows. In Sect. 2, we discuss the transcritical bifurcation analysis for the SIS epidemic network model. In Sect. 3, we propose and solve the optimal feedback mechanism for the above epidemic model. In Sect. 4, numerical simulations are presented. The paper ends up with a brief discussion.

\section{Bifurcation analysis}

In this section, we discuss the occurrence of transcritical bifurcation for system (1.1). As was pointed out in [7], since $S_{k}(t)+I_{k}(t)=1, k=1,2, \ldots, n$, we only need to present the bifurcation analysis for the following system:

$$
\left.I_{k}(t)=\lambda k(1-\beta \Theta(t))\left(1-I_{k}(t)\right) \Theta(t)-(\omega+\delta) I_{k}(t)\right), \quad k=1,2, \ldots, n .
$$

For system (2.1), there exists a disease-free equilibrium $E_{0}(\underbrace{0,0, \ldots, 0}_{n})$. We have the main result as follows.

Theorem 2.1 System (2.1) undergoes the transcritical bifurcation at $E_{0}$ when $R_{0}=1$. 
Proof The corresponding Jacobian matrix $A$ at $E_{0}$ is as follows:

$$
\left(\begin{array}{cccc}
\frac{\lambda \cdot 1 \cdot 1 \cdot P(1)}{\langle k\rangle}-(\omega+\delta) & \frac{\lambda \cdot 1 \cdot 2 \cdot P(2)}{\langle k\rangle} & \ldots & \frac{\lambda \cdot 1 \cdot n \cdot P(n)}{\langle k\rangle} \\
\frac{\lambda \cdot 2 \cdot 1 \cdot P(1)}{\langle k\rangle} & \frac{\lambda \cdot 2 \cdot 2 \cdot P(2)}{\langle k\rangle}-(\omega+\delta) & \ldots & \frac{\lambda \cdot 2 \cdot n \cdot P(n)}{\langle k\rangle} \\
\ldots & \ldots & \ldots & \ldots \\
\frac{\lambda \cdot n \cdot 1 \cdot P(1)}{\langle k\rangle} & \frac{\lambda \cdot n \cdot 2 \cdot P(2)}{\langle k\rangle} & \ldots & \frac{\lambda \cdot n \cdot n \cdot P(n)}{\langle k\rangle}-(\omega+\delta)
\end{array}\right) .
$$

Here, $\langle k\rangle=\sum_{k=1}^{n} k P(k)$ is the average degree. As was pointed out in [7], the $n$ eigenvalues of $A$ are $\lambda_{1}=\lambda_{2}=\cdots=\lambda_{n-1}=-(\delta+\omega)$ and $\lambda_{n}=(\delta+\omega)\left(R_{0}-1\right)$. One can compute that the corresponding eigenvectors are $e_{1}=\underbrace{\left(1,-\frac{P(1)}{2 P(2)}, 0, \ldots, 0\right)^{T}}_{n}, e_{2}=\underbrace{\left(1,0,-\frac{P(1)}{3 P(3)}, \ldots, 0\right)^{T}}_{n}$, $e_{n-1}=(\underbrace{1,0,0,-\frac{P(1)}{n P(n)}}_{n})^{T}$ and $e_{n}=(1,2,3, \ldots, n)^{T}$. Let $\bar{\lambda}=\lambda-\frac{(\delta+\omega)\langle k\rangle}{\left\langle k^{2}\right\rangle}, P=\left(e_{1}, e_{2}, \ldots, e_{n}\right)$, and

$$
\left(\begin{array}{l}
I_{1} \\
I_{2} \\
\cdots \\
I_{n}
\end{array}\right)=P\left(\begin{array}{l}
U_{1} \\
U_{2} \\
\cdots \\
U_{n}
\end{array}\right)
$$

We obtain the system as follows:

$$
\begin{aligned}
& \frac{d U_{k}(t)}{d t}=-(\omega+\delta) U_{k}(t)-\frac{(k+1)^{2} P(k+1)}{\langle k\rangle}\left(\bar{\lambda}+\frac{(\omega+\delta)\langle k\rangle}{\left\langle k^{2}\right\rangle}\right) \sum_{j=2}^{n}(1-j) U_{j-1} U_{n} \\
& -\frac{(k+1)^{2} P(k+1)}{P(1)\langle k\rangle}\left(\bar{\lambda}+\frac{(\omega+\delta)\langle k\rangle}{\left\langle k^{2}\right\rangle}\right)\left(-2\left\langle k^{2}\right\rangle+\left\langle k^{3}\right\rangle\right) U_{n}^{2} \\
& -2\left(\bar{\lambda}+\frac{(\omega+\delta)\langle k\rangle}{\left\langle k^{2}\right\rangle}\right) \frac{\left\langle k^{2}\right\rangle}{\langle k\rangle} U_{1} U_{n} \\
& +\frac{(k+1)^{2} P(k+1)\left\langle k^{2}\right\rangle \beta}{P(1)\langle k\rangle^{2}}\left(\bar{\lambda}+\frac{(\omega+\delta)\langle k\rangle}{\left\langle k^{2}\right\rangle}\right) \\
& \cdot\left(P(1) \sum_{j=2}^{n}(1-j) U_{j-1} U_{n}^{2}+\left\langle k^{3}\right\rangle U_{n}^{3}\right) \\
& -\frac{(k+1)^{2} P(k+1)\left\langle k^{2}\right\rangle^{2} \beta}{P(1)\langle k\rangle^{2}}\left(\bar{\lambda}+\frac{(\omega+\delta)\langle k\rangle}{\left\langle k^{2}\right\rangle}\right) \\
& \cdot\left(-\frac{P(1)}{(k+1)^{2} P(k+1)} U_{1} U_{n}^{2}+(k+1) U_{n}^{3}\right), \quad k=1,2, \ldots, n-1, \\
& \frac{d U_{n}(t)}{d t}=\frac{\left\langle k^{2}\right\rangle}{\langle k\rangle} U_{n} \bar{\lambda}+\left(\bar{\lambda}+\frac{(\omega+\delta)\langle k\rangle}{\left\langle k^{2}\right\rangle}\right) \frac{\left\langle k^{2}\right\rangle \beta}{\langle k\rangle^{2}}\left(P(1) \sum_{j=2}^{n}(1-j) U_{j-1} U_{n}^{2}+\left\langle k^{3}\right\rangle U_{n}^{3}\right) \\
& -\frac{1}{\langle k\rangle}\left(\bar{\lambda}+\frac{(\omega+\delta)\langle k\rangle}{\left\langle k^{2}\right\rangle}\right) \\
& \cdot\left(P(1) \sum_{j=2}^{n}(1-j) U_{j-1} U_{n}+\left(\frac{\left\langle k^{2}\right\rangle \alpha}{\langle k\rangle}+\left\langle k^{3}\right\rangle\right) U_{n}^{2}\right)
\end{aligned}
$$


and $\frac{d \bar{\lambda}}{d t}=0$. There exists the following center manifold $W^{c}$ :

$$
\left\{\left(U_{1}, U_{2}, \ldots, U_{n-1}\right)^{T}=h\left(U_{n}, \bar{\lambda}\right)=\left(h_{1}\left(U_{n}, \bar{\lambda}\right), h_{2}\left(U_{n}, \bar{\lambda}\right), \ldots, h_{n-1}\left(U_{n}, \bar{\lambda}\right)\right)^{T}\right\}
$$

Since $h(0,0)=0, h_{U_{n}}(0,0)=\frac{\partial h}{\partial U_{n}}(0,0)=0$, and $h_{\bar{\lambda}}(0,0)=\frac{\partial h}{\partial \bar{\lambda}}(0,0)=0$, and by use of the Taylor expansion of $h\left(U_{n}, \bar{\lambda}\right)$, we can suppose that $U_{k}=d_{k} U_{n}^{2}+e_{k} U_{n} \bar{\lambda}+f_{k} \bar{\lambda}^{2}, k=1,2, \ldots, n-1$. Here, the coefficients $d_{k}, e_{k}, f_{k}$ will be determined later. Actually, putting the above equalities about $U_{k}$ into (2.2) and (2.3), through complicated computing, we can obtain

$$
\left(\begin{array}{c}
U_{1} \\
U_{2} \\
\cdots \\
U_{n-1}
\end{array}\right)=\left(\begin{array}{c}
\frac{2^{2} P(2)(\delta+\omega)\left(2\left\langle k^{2}\right\rangle-\left\langle k^{3}\right\rangle\right)}{P(1)\left(k^{3}\right\rangle} U_{n}^{2}+o\left(U_{n}^{3}\right) \\
\frac{3^{2} P(3)(\delta+\omega)\left(3\left\langle k^{2}\right\rangle-\left\langle k^{3}\right\rangle\right)}{P(1)\left\langle k^{3}\right\rangle} U_{n}^{2}+o\left(U_{n}^{3}\right) \\
\ldots \\
\frac{n^{2} P(n)(\delta+\omega)\left(n\left\langle k^{2}\right\rangle-\left\langle k^{3}\right\rangle\right)}{P(1)\left\langle k^{3}\right\rangle} U_{n}^{2}+o\left(U_{n}^{3}\right)
\end{array}\right) .
$$

As a result, from (2.3) and (2.4), system (2.1) reduced on the center manifold reads as follows:

$$
\frac{d U_{n}(t)}{d t}=\frac{\left\langle k^{2}\right\rangle}{\langle k\rangle} U_{n} \bar{\lambda}-(\omega+\delta)\left(\frac{\beta}{\langle k\rangle}+\frac{\left\langle k^{3}\right\rangle}{\left\langle k^{2}\right\rangle}\right) U_{n}^{2}+o\left(U_{n}^{3}\right) \triangleq G\left(U_{n}, \bar{\lambda}\right) .
$$

Since $G(0,0)=0, G_{U_{n}}(0,0)=0, G_{\bar{\lambda}}(0,0)=0$, and $G_{U_{n}, \bar{\lambda}}^{2}-G_{U_{n}, U_{n}} G_{\bar{\lambda}, \bar{\lambda}}=\frac{\left\langle k^{2}\right\rangle^{2}}{\langle k\rangle^{2}} \neq 0$. Thus the proof is completed.

Remark 2.1 We also can determine the direction of the bifurcation in Theorem 1 as follows. For system (2.1), the endemic equilibrium should satisfy the following equation:

$$
\frac{1}{\langle k\rangle} \sum_{h=1}^{n} \frac{\lambda h^{2} p(h)}{\frac{\delta+\omega}{1-\beta \Theta}+\lambda h \Theta}=1 .
$$

Since $R_{0}=\frac{\lambda\left\langle k^{2}\right\rangle}{(\delta+\omega)(k\rangle}$, we multiply the denominator and numerator of (2.6) by $\frac{\left\langle k^{2}\right\rangle}{(\delta+\omega)\langle k\rangle}$. Then we can obtain the following equality:

$$
\frac{1}{\langle k\rangle} \sum_{h=1}^{n} \frac{R_{0} h^{2} p(h)}{\frac{\left\langle k^{2}\right\rangle}{\langle k\rangle(1-\beta \Theta)}+R_{0} h \Theta}=1 .
$$

Consequently, according to (2.7), the derivative of $\Theta$ with respect to $R_{0}$ at the critical value $\left(R_{0}, \Theta\right)=(1,0)$ is $\left.\frac{\partial \Theta}{\partial R_{0}}\right|_{\left(R_{0}, \Theta\right)=(1,0)}=\frac{\left\langle k^{2}\right\rangle^{2}}{\delta\left\langle k^{2}\right\rangle^{2}+\langle k\rangle^{2}\left\langle k^{3}\right\rangle}>0$. So the endemic equilibrium curve bifurcates forward. In other words, system (2.1) exhibits forward bifurcation at $R_{0}=1$.

Remark 2.2 In system (2.5), let $\bar{\lambda}=0$, we have

$$
\frac{d U_{n}(t)}{d t}=-(\omega+\delta)\left(\frac{\beta}{\langle k\rangle}+\frac{\left\langle k^{3}\right\rangle}{\left\langle k^{2}\right\rangle}\right) U_{n}^{2}+o\left(U_{n}^{3}\right)
$$

So $E_{0}$ is stable when $R_{0}=1$. 
Remark 2.3 For the epidemic model on networks, most existing literature works only investigate the two cases, i.e., $R_{0}>1$ and $R_{0}<1$. In Theorem 2.1, Remark 2.1, and Remark 2.2, we pay attention to the critical case $R_{0}=1$ and present the strict theoretical proof for the existence of the transcritical bifurcation. Thus, the above results improve and supplement the corresponding results in [7] and [29,30].

\section{The optimal control analysis}

As we know, in reality, when there exists a disease spreading in a crowd and the number of infections is increasing, people will decrease the contact with others consciously. As a result, the above conscious behavior can naturally reduce the number of the infected. On the contrary, when the number of the infected is going down, people interact with others more closely. In other words, people's self-awareness has a direct effect on the spread of a disease. So analyzing the fear factor or the feedback mechanism coefficient is very interesting and indispensable. In this section, we pay attention to discussing the optimal fear factor or the optimal feedback mechanism coefficient. According to the optimal parameter, the media can report the disease's severity suitably, which not only reduces contact with other people but also avoids the panic in public.

In this section, we use the optimal control theory in [31] to analyze the optimal fear factor strategy. One of our goals is to reduce the number of the infected as much as possible. The other goal is that the fear factor is as low as possible. In detail, we discuss the optimal control problem to minimize the objective functional

$$
J(u(\cdot))=\int_{0}^{T} \sum_{k=1}^{n}\left[I_{k}(t)+\frac{1}{2} c_{k}^{2} u_{k}^{2}(t)\right] d t
$$

subject to

$$
\left\{\begin{array}{l}
\frac{d S_{k}(t)}{d t}=\delta-\delta S_{k}(t)-\lambda k\left(1-u_{k}(t) \Theta(t)\right) S_{k}(t) \Theta(t)+\omega I_{k}(t), \\
\frac{d I_{k}(t)}{d t}=\lambda k\left(1-u_{k}(t) \Theta(t)\right) S_{k}(t) \Theta(t)-(\omega+\delta) I_{k}(t), \quad k=1,2, \ldots, n .
\end{array}\right.
$$

Here, $c_{k}, k=1,2, \ldots, n$, are positive weight constants to keep a balance between the size of $I_{k}(t)$ and the fear factor $u_{k}(t)$. Different from the constant coefficient $\beta$ in (1.1), the fear factor $u_{k}(t)$ in (3.2) is time-dependent since we think that the individual has different perception of disease as time goes on. Since the fear factor or the feedback mechanism coefficient $u_{k}(t)$ is related to the media's propaganda for disease, it is natural to have a restriction on the control variable such that $0 \leq u_{k}(t) \leq 1$. In other words, for the optimal control problem (3.1)-(3.2), the admissible control set is $\mathcal{U}_{a d}=\left\{u_{k}(\cdot) \in L^{2}[0, T], 0 \leq\right.$ $\left.u_{k}(t) \leq 1, t \in[0, T]\right\}$. Here we have to point out that the reasons why we choose the objective function $J$ as (3.1) are as follows. On the one hand, both the government and the public wish that the people's fear factor coefficient is as low as possible when the epidemic outbreaks. On the other hand, we also expect that the number of infected is as small as possible until the disease completely disappears. Firstly, we present the existence of the optimal control strategy as follows.

Theorem 3.1 There exists an optimal control $u^{*}(t)$ such that $J\left(u^{*}(\cdot)\right)=\min _{u \in U_{a d}^{n}} J(u(t))$ subject to the performance index (3.1) and the controlled system (3.2). 
Proof We can prove that the following conditions are satisfied:

(i) System (3.2) is solvable for any control variable $u \in U_{a d}^{n}$. In fact, the right-hand side of the state system is continuously differentiable and the claim follows from the continuation theorem for differential equations [32].

(ii) $U_{a d}^{n}$ is convex. In fact, let $u=\left(u_{1}(\cdot), u_{1}(\cdot), \ldots, u_{n}(\cdot)\right) \in U_{a d}^{n}$, $\bar{u}=\left(\bar{u}_{1}(\cdot), \bar{u}_{1}(\cdot), \ldots, \bar{u}_{n}(\cdot)\right) \in U_{a d}^{n}$, and $0 \leq p \leq 1$. We can have $0 \leq(1-p) u_{i}(t)+p \bar{u}_{i}(t) \leq 1$ since $0 \leq u_{i}(t), \bar{u}_{i}(t) \leq 1(i=1,2, \ldots, n)$. As a result, $(1-p) u+p \bar{u} \in U_{a d}^{n}$. The above gives that $U_{a d}^{n}$ is convex.

(iii) $U_{a d}^{n}$ is closed. In fact, let $u=\left(u_{1}(\cdot), u_{1}(\cdot), \ldots, u_{n}(\cdot)\right)$ be a limit point of $U_{a d}^{n}$. And let $u^{(m)}=\left(u_{1}^{(m)}(\cdot), u_{1}^{(m)}(\cdot), \ldots, u_{n}^{(m)}(\cdot)\right) \in U_{a d}^{n}, m=1,2, \ldots$, be a sequence of points in $U_{a d}^{n}$ such that $\left\|u_{i}^{(m)}(\cdot)-u_{i}(\cdot)\right\|=\left[\int_{0}^{T}\left|u_{i}^{(m)}(t)-u_{i}(t)\right|^{2}\right]^{\frac{1}{2}} \leq \frac{1}{m}$. Due to the completeness of $L^{2}[0, T]^{n}$, we have $u(\cdot)=\lim _{m \rightarrow \infty} u^{(m)}(\cdot) \in L^{2}[0, T]^{n}$. Also, $0 \leq u_{i}(t)=\lim _{m \rightarrow \infty} u_{i}^{(m)}(t) \leq 1(i=1,2, \ldots, n)$. This leads to the closeness of $U_{a d}^{n}$.

(iv) The right-hand side of the state system is bounded by a linear function in the state variable. In fact, let $f_{1}(S, I)=\delta-\delta S_{k}(t)-\lambda k\left(1-u_{k}(t) \Theta(t)\right) S_{k}(t) \Theta(t)+\omega I_{k}(t)$ and $f_{2}(S, I)=\lambda k\left(1-u_{k}(t) \Theta(t)\right) S_{k}(t) \Theta(t)-(\omega+\delta) I_{k}(t)$. We have

$$
\delta-(\delta+n \lambda) S_{k}(t)+\omega I_{k}(t) \leq f_{1}(S, I) \leq \delta-\delta S_{k}(t)+\omega I_{k}(t)
$$

and

$$
-(\delta+n \lambda) I_{k}(t) \leq f_{2}(S, I) \leq \lambda n S_{k}(t)-(\delta+n \lambda) I_{k}(t)
$$

(v) The Lagrangian $L(I, u)=\sum_{k=1}^{n} I_{k}(t)+\frac{c_{k}}{2} u_{k}^{2}(t)$ is convex on $U_{a d}^{n}$. In fact, let $u_{1}, u_{2} \in U_{a d}^{n}$ and $0 \leq \theta \leq 1$, we have

$$
\begin{aligned}
& L\left(I,(1-\theta) u_{1}+\theta u_{2}\right) \\
& \quad=\sum_{k=1}^{n}\left[I_{k}(t)+\frac{c_{k}}{2}\left((1-\theta) u_{1}(t)+\theta u_{2}(t)\right)^{2}\right] \\
& \quad \leq(1-\theta) \sum_{k=1}^{n}\left[I_{k}(t)+\frac{c_{k}}{2} u_{1 k}^{2}(t)\right)+\theta \sum_{k=1}^{n}\left[I_{k}(t)+\frac{c_{k}}{2} u_{2 k}^{2}(t)\right) \\
& \quad=(1-\theta) L\left(I, u_{1}\right)+\theta L\left(I, u_{2}\right) .
\end{aligned}
$$

(vi) There exist constants $\rho>1, c_{1}>0, c_{2}$ such that $L \geq c_{1}\|u\|^{\rho}+c_{2}$. In fact, the Lagrangian $L$ satisfies $L \geq \frac{1}{2} \min _{1 \leq k \leq n} c_{k}^{2}\|u\|^{2}$.

By utilizing the corresponding results in $[31,33,34]$, the above completes the existence of an optimal control.

Remark 3.1 The uniqueness of the optimal control can be obtained by similar analysis in Fister et al. [35].

Secondly, we investigate the optimal solution by the Pontryagin minimum principle [31]. Define the Hamiltonian $H$ as

$$
H=L+\sum_{k=1}^{n}\left[\lambda_{1 k}(t) \frac{d S_{k}(t)}{d t}+\lambda_{2 k}(t) \frac{d I_{k}(t)}{d t}\right]
$$


Here, $\lambda_{1 k}$ and $\lambda_{2 k}$ are the adjoint variables to be determined later. Then, applying the necessary conditions to the Hamiltonian $H$, we can obtain the main result as follows.

Theorem 3.2 Let $S_{k}^{*}(t)$ and $I_{k}^{*}(t)$ be optimal state solutions with associated optimal control variable $u_{k}^{*}(t)$ for the optimal control problem (3.1) and (3.2). Then, the adjoint variables $\lambda_{1 k}(t)$ and $\lambda_{2 k}(t)$ satisfy

$$
\begin{aligned}
\frac{d \lambda_{1 k}}{d t}= & \left(\delta+\lambda k\left(1-u_{k}^{*}(t) \Theta(t)\right) \Theta(t)\right) \lambda_{1 k}(t)-\lambda k\left(1-u_{k}^{*}(t) \Theta(t)\right) \Theta(t) \lambda_{2 k}(t), \\
\frac{d \lambda_{2 k}}{d t}= & -1-\omega \lambda_{1 k}(t)+(\omega+\delta) \lambda_{2 k}(t) \\
& -\frac{\lambda k p(k)}{\langle k\rangle} \sum_{i=1}^{n}\left(\lambda_{1 i}(t)-\lambda_{2 i}(t)\right) i\left(1-2 u_{i}^{*}(t) \Theta(t)\right) S_{i}^{*}(t), \quad k=1,2, \ldots, n .
\end{aligned}
$$

The transversality conditions are

$$
\lambda_{1 k}(T)=\lambda_{2 k}(T)=0 .
$$

Furthermore, the optimal control is given by

$$
u_{k}^{*}(t)=\min \left\{\max \left(0, \frac{\lambda k S_{k}^{*}(t) \Theta^{2}(t)}{c_{k}}\left(\lambda_{2 k}(t)-\lambda_{1 k}(t)\right)\right), 1\right\} .
$$

Proof We will deduce the adjoint equations and the transversality conditions by use of the necessary condition for the optimal control problems which can be found in [31]. Setting $S_{k}(t)=S_{k}^{*}(t), I_{k}(t)=I_{k}^{*}(t)$ and differentiating Hamiltonian (3.3) with respect to $S_{k}$ and $I_{k}$, we can obtain

$$
\begin{aligned}
& \frac{d \lambda_{1 k}}{d t}=-\left.\frac{\partial H}{\partial S_{k}}\right|_{\left(S_{k}(t), I_{k}(t), u_{k}(t)=\left(S_{k}^{*}(t), I_{k}^{*}(t), u_{k}^{*}(t)\right)\right.}, \\
& \frac{d \lambda_{2 k}}{d t}=-\left.\frac{\partial H}{\partial I_{k}}\right|_{\left(S_{k}(t), I_{k}(t), u_{k}(t)=\left(S_{k}^{*}(t), I_{k}^{*}(t), u_{k}^{*}(t)\right)\right.} .
\end{aligned}
$$

Through direct calculation, it is easy to obtain Eqs. (3.4). Also, by the optimal condition, we have

$$
\frac{\partial H}{\partial u_{k}}=c_{k} u_{k}^{*}(t)+\lambda k S_{k}^{*}(t) \Theta^{2}(t) \lambda_{1 k}(t)-\lambda k S_{k}^{*}(t) \Theta^{2}(t) \lambda_{2 k}(t)=0 .
$$

It follows that $u_{k}^{*}(t)=\frac{\lambda k s_{k}^{*}(t) \Theta^{2}(t)}{c_{k}}\left(\lambda_{2 k}(t)-\lambda_{1 k}(t)\right)$. Using the property of the control space, we obtain the following restriction condition:

$$
u_{k}^{*}= \begin{cases}0, & \text { if } \frac{\lambda S_{k}^{*}(t) \Theta^{2}(t)}{c_{k}}\left(\lambda_{2 k}(t)-\lambda_{1 k}(t)\right) \leq 0, \\ \frac{\lambda k S_{k}^{*}(t)}{c_{k}}\left(\lambda_{2 k}(t)-\lambda_{1 k}(t)\right) \Theta^{2}(t), & \text { if } 0<\frac{\lambda k S_{k}^{*}(t) \Theta^{2}(t)}{c_{k}}\left(\lambda_{2 k}(t)-\lambda_{1 k}(t)\right)<1, \\ 1, & \text { if } \frac{\lambda k S_{k}^{*}(t) \Theta^{2}(t)}{c_{k}}\left(\lambda_{2 k}(t)-\lambda_{1 k}(t)\right) \geq 1 .\end{cases}
$$

So we have (3.6), which is the optimal control $u_{k}^{*}(t)$ in compact notation. Thus we complete the proof. 
Lastly, we can obtain the optimality system as follows:

$$
\left\{\begin{aligned}
\frac{d S_{k}^{*}(t)}{d t}= & -\lambda k\left(1-\min \left\{\max \left(0, \frac{\lambda k S_{k}^{*}(t) \Theta^{2}(t)}{c_{k}}\left(\lambda_{2 k}(t)-\lambda_{1 k}(t)\right)\right), 1\right\} \Theta(t)\right) \\
& \cdot S_{k}^{*}(t) \Theta(t)+\omega I_{k}^{*}(t)+\delta-\delta S_{k}^{*}(t) \\
\frac{d I_{k}^{*}(t)}{d t}= & \lambda k\left(1-\min \left\{\max \left(0, \frac{\lambda k S_{k}^{*}(t) \Theta^{2}(t)}{c_{k}}\left(\lambda_{2 k}(t)-\lambda_{1 k}(t)\right)\right), 1\right\} \Theta(t)\right) \\
& \cdot S_{k}^{*}(t) \Theta(t)-(\omega+\delta) I_{k}^{*}(t) \\
\frac{d \lambda_{1 k}}{d t}= & \delta \lambda_{1 k}(t)+\left(1-\min \left\{\max \left(0, \frac{\lambda k S_{k}^{*}(t) \Theta^{2}(t)}{c_{k}}\left(\lambda_{2 k}(t)-\lambda_{1 k}(t)\right)\right), 1\right\} \Theta(t)\right) \\
& \cdot \lambda k \Theta(t)\left(\lambda_{1 k}(t)-\lambda_{2 k}(t)\right), \\
\frac{d \lambda_{2 k}}{d t}= & -1-\omega \lambda_{1 k}(t)+(\omega+\delta) \lambda_{2 k}(t)-\frac{\lambda k p(k)}{\langle k\rangle} \sum_{i=1}^{n}\left(\lambda_{1 i}(t)-\lambda_{2 i}(t)\right) i \\
& \cdot\left(1-2 \min \left\{\max \left(0, \frac{\lambda i S_{i}^{*}(t)}{c_{i}}\left(\lambda_{2 i}(t)-\lambda_{1 i}(t)\right) \Theta^{2}(t)\right), 1\right\} \Theta(t)\right) S_{i}^{*}(t)
\end{aligned}\right.
$$

\section{Numerical simulations}

In this section, we present numerical simulation. Here all the parameter values are chosen hypothetically due to the unavailability of real world data. Let $\delta=0.1, \omega=0.1$ and $c_{k}=0.5$, $k=1, \ldots, 500$. Here the network size $n$ is 500 and the heterogeneous structure is characterized by a BA network and a WS small-world network, respectively. The above BA network is produced as follows. Four initial nodes are fully connected and then a new node with three new edges at each time step is added. The WS small-world network is generated with probability 0.1 for rewiring links. Here, each node is symmetrically connected with its six nearest neighbors in its initial nearest neighbor network. For the BA network, in cases (a), (b), and (c) of Fig. 1, we plot the trajectories of the number of the average infected $I=\sum_{k=1}^{n} p(k) I_{k}(t)$ when $R_{0}<1, R_{0}=1$, and $R_{0}>1$, respectively. For the WS small-world network, the corresponding simulation is presented in Fig. 2. In Figs. $3-6$, let $\lambda=0.2$, we shall achieve sub-figures (a) and (b) on the BA network and the WS small-world network, respectively.

In order to show the effectiveness of the proposed optimal control strategy, we make a comparison between several constant controls and optimal control. For example, from Fig. 3, one can see that the optimal control can make the average number of the infected relatively small compared to other five constant control strategies. In Fig. 4, we draw the dynamical curve of the optimal control variables $u_{k}^{*}(t)$ for different degree $k$. One can notice that $u_{k}^{*}(t)$ converges to zero, which coincides with (3.5). Comparing (a) and (b), we find that in the early stage of epidemic outbreak the control strength on the BA network should be larger than that on the WS small-world network. As time goes on, the control

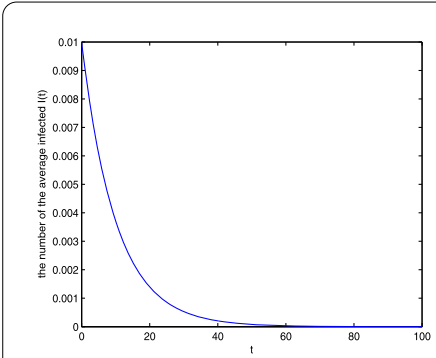

(a)

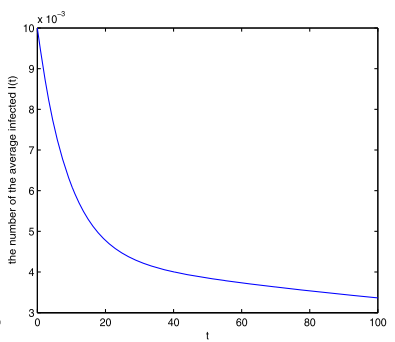

(b)

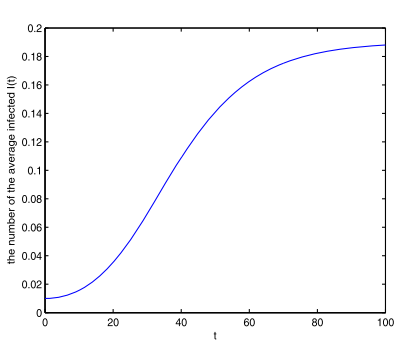

(c)

Figure 1 The average infected densities $I=\sum_{k=1}^{n} p(k) I_{k}(t)$ with respect to $(\mathbf{a}) R_{0}<1$, (b) $R_{0}=1$, and (c) $R_{0}>1$ on the BA network 


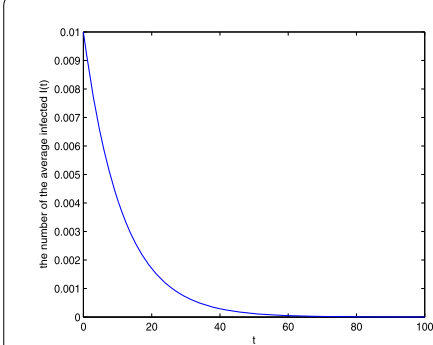

(a)

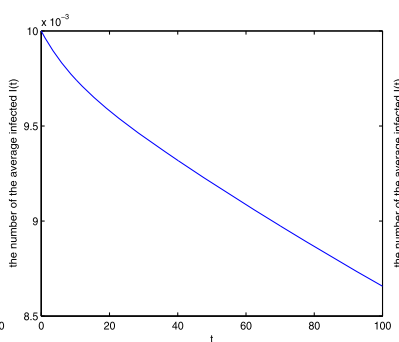

(b)

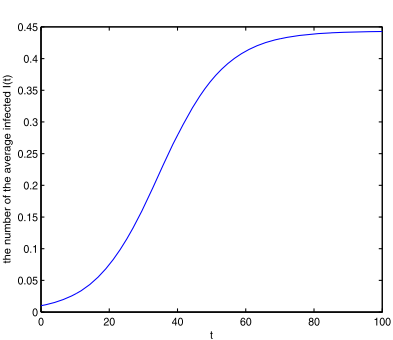

(c)

Figure 2 The average infected densities $I=\sum_{k=1}^{n} p(k) I_{k}(t)$ with respect to (a) $R_{0}<1$, (b) $R_{0}=1$, and (c) $R_{0}>1$ on the WS small-world network

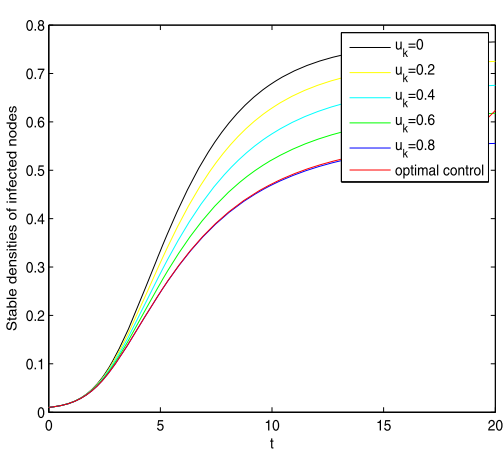

(a)

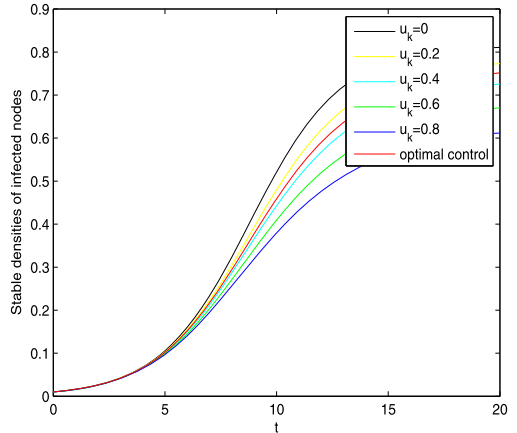

(b)

Figure 3 The average infected densities $I=\sum_{k=1}^{n} p(k) I_{k}(t)$ with respect to five kinds of constant control $u_{k}(t)=u$ and the optimal control $u_{k}^{*}(t)$

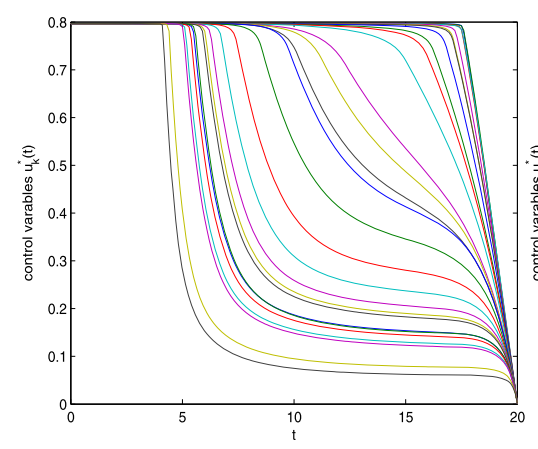

(a)

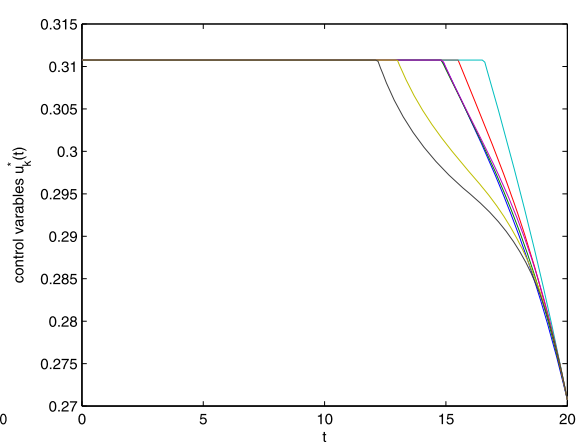

(b)

Figure 4 The dynamical process of the optimal control variables $u_{k}^{*}(t), k=1,2, \ldots, n$

strength on the BA network is lower than that on the WS small-world network. The reason may be that people contact with others closely in the BA network. So it is important to control the epidemic as soon as possible, which results in great control strength in the early stage of epidemic outbreak. Once the epidemic is under control in the BA network, a small amount of control strength is consequently needed since the nodes with large degree occupy a small fraction of the network size. Moreover, from Fig. 5, one can find that the 


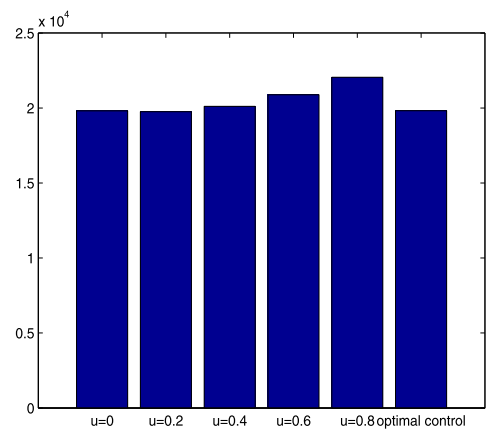

(a)

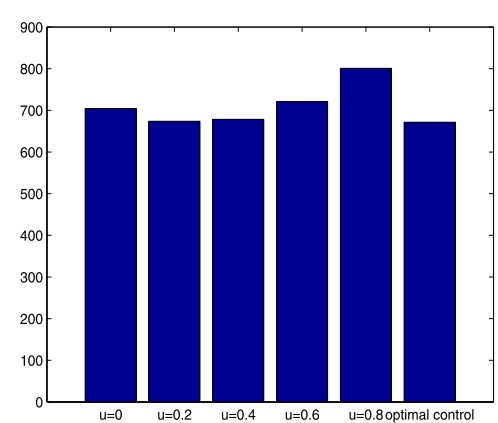

(b)

Figure 5 The values of the performance index $J$ when $u_{k}(t)=0,0.2,0.4,0.6,0.8$ and optimal control $u_{k}^{*}(t)$

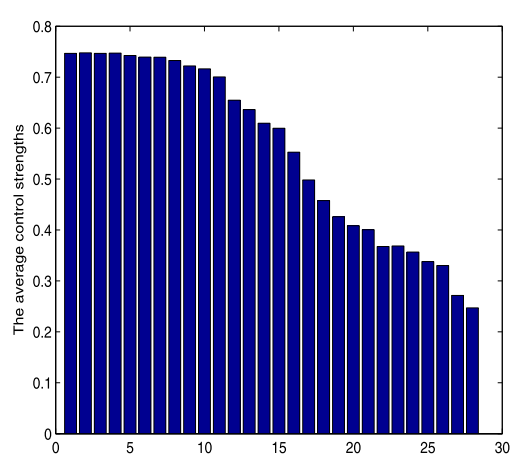

(a)

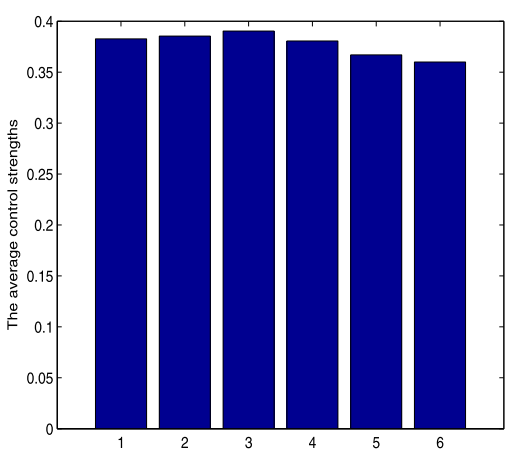

(b)

Figure 6 The average control strength $\bar{u}_{k}, k=1,2, \ldots, n$

optimal control strategy indeed makes the objective function $J$ achieve the minimal value, which is consistent with the theoretical results.

Lastly, we explore the average optimal control strength $\bar{u}_{k}=\int_{0}^{T} u_{k}^{*}(t) d t$ in Fig. 6. One can find that on the BA network, those nodes with small degree should be strengthened in order to control the epidemic and achieve the minimum performance index. Maybe it is because those nodes with small degree often do not have a clear understanding for the severity of the disease. So the public or the media should advise them to decrease the communication with others. However, on the WS small-world network, there is little difference in the average control strength for the nodes with different degree. Maybe it is because the degree of most of the nodes is equal to 6, i.e., the topological structure of the WS network is relatively homogenous. So most of the nodes should be strengthened. The main results in this paper are a good extension and supplement to those in [7, 29], and [30]. All the above simulation analysis may provide the public some guidance when the whole society faces up to infectious diseases.

\section{Conclusion}

In this paper, we have discussed an SIS epidemic model with feedback mechanism on heterogeneous networks. The feedback mechanism is incorporated in two manners, i.e., constant control and time-varying control. Firstly, for the constant feedback mechanism, the existence of the transcritical bifurcation when $R_{0}=1$ is strictly proved in Theorem 2.1. 
And we have shown that the direction of bifurcation is forward. Secondly, in order to further analyze the influence of the feedback mechanism on the epidemic spreading, for the time-varying control, we have proposed the optimal control problem which minimizes the number of the infected and the cost associated with the feedback mechanism. In Theorem 3.1 and Theorem 3.2, we have obtained the existence and uniqueness of the optimal control strategy.

Finally, we have performed the numerical simulation to illustrate our theoretical results. Especially, a comparison between optimal control and other constant controls is presented in Figs. 3-5. It is easy to see that the optimal control is much more effective for reducing the number of infected individuals and minimizing the performance index. Furthermore, Fig. 6 reveals that the optimal control strategy is closely related to the topology of the network. In detail, those individuals with small degree should be strengthened on the BA network and the control strength is more balanced for the nodes with different degree on the WS network. All the results in this paper are a good extension and supplement to those in [7, 29], and [30]. The above analysis will have certain guidance when analyzing and controlling the disease spread.

\section{Funding}

This work was supported by the National Natural Science Foundation of China under Grant 11601085, the Natural Science Foundation of Fujian Province under Grant $2017 \mathrm{~J} 01400$ and 2018J01664, the Scientific Research Foundation of Fuzhou University under Grant GXRC-17026 and GXRC-18047.

\section{Competing interests}

The authors declare that there is no conflict of interests.

\section{Authors' contributions}

All authors contributed equally to the writing of this paper. All authors read and approved the final manuscript.

\section{Publisher's Note}

Springer Nature remains neutral with regard to jurisdictional claims in published maps and institutional affiliations.

Received: 12 July 2019 Accepted: 10 December 2019 Published online: 19 December 2019

\section{References}

1. Zhang, Y.X., Li, K.Z.: Successive lag synchronization on nonlinear dynamical networks via a periodically intermittent control. Nonlinear Dyn. 95, 3075-3089 (2019)

2. Li, K.Z., Zhang, H.F., Zhu, G.H., Small, M., Fu, X.C.: Suboptimal control and targeted constant control for semi-random epidemic networks. IEEE transctions on systems, man and cybernetics: systems, accepted

3. Liu, M.X., Chang, Y.T., Wang, H.Y., Li, B.X.: Dynamics of the impact of Twitter with time delay on the spread of infectious diseases. Int. J. Biomath. 11, 1850067 (2018)

4. Liu, M.X., Wu, J., Sun, Y.-Z.: Adaptive finite-time outer synchronization between two complex dynamical networks with noise perturbation. Nonlinear Dyn. 89, 2967-2977 (2017)

5. Huang, S.Y., Chen, F.D., Zhang, Y.H.: Global analysis of epidemic spreading with a general feedback mechanism on complex networks. Adv. Differ. Equ. 2019, 154 (2019). http://doi.org/10.1186/s13662-019-2095-3

6. Pastor-Satorras, R., Vespignani, A.: Epidemic spreading in scale-free networks. Phys. Rev. Lett. 86, 3200-3203 (2001)

7. Zhang, J.C., Sun, J.T.: Stability analysis of an SIS epidemic model with feedback mechanism on networks. Phys. A, Stat. Mech. Appl. 394, 24-32 (2014)

8. Kang, H.Y., Fu, X.C.: Epidemic spreading and global stability of an SIS model with an infective vector on complex networks. Commun. Nonlinear Sci. Numer. Simul. 27, 30-39 (2015)

9. Li, C.-H.: Dynamics of a network-based SIS epidemic model with nonmonotone incidence rate. Phys. A, Stat. Mech. Appl. 427, 234-243 (2015)

10. Wei, X.D., Liu, L.J., Zhou, W.S.: Global stability and attractivity of a network-based SIS epidemic model with nonmonotone incidence rate. Phys. A, Stat. Mech. Appl. 469, 789-798 (2017)

11. Kang, H.Y., Lou, Y.J., Chen, G.R., Chu, S., Fu, X.C.: Epidemic spreading and global stability of a new SIS model with delay on heterogeneous networks. J. Biol. Syst. 23, 577-595 (2015)

12. Zhang, J.P., Yang, C., Jin, Z., Li, J.: Dynamics analysis of SIR epidemic model with correlation coefficients and clustering coefficient in networks. J. Theor. Biol. 449, 1-13 (2018)

13. Huo, J.J., Zhao, H.Y.: Dynamical analysis of a fractional SIR model with birth and death on heterogeneous complex networks. Phys. A, Stat. Mech. Appl. 448, 41-56 (2016)

14. Chen, L.J., Sun, J.T.: Global stability and optimal control of an SIRS epidemic model on heterogeneous networks. Phys. A, Stat. Mech. Appl. 410, 196-204 (2014) 
15. Chen, L.J., Sun, J.T.: Optimal vaccination and treatment of an epidemic network model. Phys. Lett. A 378, 3028-3036 (2014)

16. Huang, S.Y.: Global dynamics of a network-based WSIS model for mobile malware propagation over complex networks. Phys. A, Stat. Mech. Appl. 503, 293-303 (2018)

17. Huang, S.Y., Chen, F.D., Chen, L.J.: Global dynamics of a network-based SIQRS epidemic model with demographics and vaccination. Commun. Nonlinear Sci. Numer. Simul. 43, 296-310 (2017)

18. Wanduku, D.: Complete global analysis of a two-scale network SIRS epidemic dynamic model with distributed delay and random perturbations. Appl. Math. Comput. 294, 49-76 (2017)

19. Krause, A.L., Kurowski, L., Yawar, K., Van Gorder, R.A.: Stochastic epidemic metapopulation models on networks: SIS dynamics and control strategies. J. Theor. Biol. 449, 35-52 (2018)

20. Wang, X.H., Wang, Z., Shen, H.: Dynamical analysis of a discrete-time SIS epidemic model on complex networks. Appl. Math. Lett. 94, 292-299 (2019)

21. Huang, Y.-J., Li, C.-H.: Backward bifurcation and stability analysis of a network-based SIS epidemic model with saturated treatment function. Phys. A, Stat. Mech. Appl. 527, 121407 (2019)

22. Li, C.-H., Yousef, A.M.: Bifurcation analysis of a network-based SIR epidemic model with saturated treatment function. Chaos: an interdisciplinary. J. Nonlinear Sci. 29, 033129 (2019)

23. Xu, D.G., Xu, X.Y., Xie, Y.F., Yang, C.H.: Optimal control of an SIVRS epidemic spreading model with virus variation based on complex networks. Commun. Nonlinear Sci. Numer. Simul. 48, 200-210 (2017)

24. Hu, P., Ding, L., Hadzibeganovic, T.: Individual-based optimal weight adaptation for heterogeneous epidemic spreading networks. Commun. Nonlinear Sci. Numer. Simul. 63, 339-355 (2018)

25. Yang, L.-X., Draief, M., Yang, X.F.: The optimal dynamic immunization under a controlled heterogeneous node-based SIRS model. Phys. A, Stat. Mech. Appl. 450, 403-415 (2018)

26. Liu, L., Luo, X.F., Chang, L.L.: Vaccination strategies of an SIR pair approximation model with demographics on complex networks. Chaos Solitons Fractals 104, 282-290 (2017)

27. Feng, Y., Ding, L., Hu, P.: Epidemic spreading on random surfer networks with optimal interaction radius. Commun. Nonlinear Sci. Numer. Simul. 56, 344-353 (2018)

28. Zhang, J.C., Sun, J.T.: Analysis of epidemic spreading with feedback mechanism in weighted networks. Int. J. Biomath. 08, 1550007 (2015). https://doi.org/10.1142/s1793524515500072

29. Li, T., Liu, X.D., Wu, J., Wan, C., Guan, Z.-H., Wang, Y.M.: An epidemic spreading model on adaptive scale-free networks with feedback mechanism. Phys. A, Stat. Mech. Appl. 450, 649-656 (2016)

30. Wei, X.D., Xu, G.C., Zhou, W.S.: Global stability of an SIS epidemic model with feedback mechanism on networks. Adv. Differ. Equ. 2018, 60 (2018). https://doi.org/10.1186/s13662-018-1501-6

31. Lenhart, S., Workman, J.T.: Optimal control applied to biological models. In: Mathematical and Computational Biology Series. Chapman \& Hall, London (2007)

32. Robinson, R.C.: An Introduction to Dynamical Systems: Continuous and Discrete. Prentice Hall, New York (2012)

33. Kamien, M.I., Schwartz, N.L.: Dynamic Optimization: The Calculus of Variations and Optimal Control in Economics and Management. Elsevier, Amsterdam (2000)

34. Fleming, W.H., Rishel, R.W.: Deterministic and Stochastic Optimal Control. Springer, New York (1975)

35. Fister, K.R., Lenhart, S., Mcnally, J.S.: Optimizing chemotherapy in an HIV model. Electron. J. Differ. Equ. 32, 1-12 (1998)

\section{Submit your manuscript to a SpringerOpen ${ }^{\circ}$ journal and benefit from:}

- Convenient online submission

- Rigorous peer review

- Open access: articles freely available online

- High visibility within the field

- Retaining the copyright to your article

Submit your next manuscript at $\boldsymbol{s p r i n g e r o p e n . c o m ~}$ 